INPLASY

PROTOCOL

To cite: Wan et al. The ability of national early warning scores to predict mortality among patients in the prehospital setting: a meta-analysis. Inplasy protocol 202220109. doi:

10.37766/inplasy2022.2.0109

Received: 24 February 2022

Published: 24 February 2022

Corresponding author:

Wan Wenlin

362419144@qq.com

Author Affiliation:

People's Hospital of Leshan.

Support: Not applicable.

Review Stage at time of this submission: The review has not yet started.

Conflicts of interest:

None declared.

\section{The ability of national early warning scores to predict mortality among patients in the prehospital setting:} a meta-analysis

Wan, WL1; Li, LX2; Ou, O3; Chen, B4; Ma, ZQ5; Yuan, P6.

Review question / Objective: We want to evaluate the ability of national early warning scores to predict short-term mortality in patients in the prehospital setting.

Eligibility criteria: Inclusion criteria (1) The study recruited adult patients ( $\geq 16$ years old) in the prehospital setting; (2) The study applied the NEWS or NEWS2; (3) Patients transported by ambulance in the prehospital setting; (4) The study outcomes of interest were admission to ICU, short-term mortality or sepsis; Exclusion criteria: (1) The study population was children or pregnant; (2)Helicopter emergency service; (3)If studies used the same database, we included the study with the most patients and excluded the others.

INPLASY registration number: This protocol was registered with the International Platform of Registered Systematic Review and Meta-Analysis Protocols (INPLASY) on 24 February 2022 and was last updated on 24 February 2022 (registration number INPLASY202220109).

\section{INTRODUCTION}

Review question / Objective: We want to evaluate the ability of national early warning scores to predict short-term mortality in patients in the prehospital setting.

Condition being studied: Early warning scores(EWS) can help determine clinical deterioration and prevent adverse events. Among all the EWS, National early warning
Score (NEWS) was developed by the Royal College of Physicians in 2012 and updated in 2017 is now widely used internationally. NEWS was composed of seven physiological parameters: respiratory rate, whether to use oxygen (FiO2), oxygen saturation (SpO2), blood pressure, heart rate, consciousness level (using AVPU tool) and body temperature. Royal College of Physicians(2012) suggests that NEWS can be used in the pre-hospital assessment of acute patients by "first responders" (such 
as community hospitals, ambulance services and primary care) to discern and promote the evaluate and to predict the severity of the illness to the receiving hospital for triage. The pre-hospital environment refers to the care provided before or during the emergency services take the patient to the hospital. Therefore, the environment is quite complex and the patient population is mixed with diseases. Although there have been some studies on the use of early warning scores (NEWS) in prehospital settings to predict patient mortality and deterioration, it is not clear whether they are the best research evidence to assist health care providers in making the best decisions for patients.

\section{METHODS}

Search strategy: Pubmed=

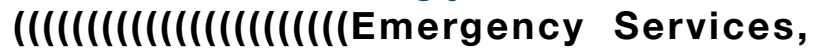
Medical[Title/Abstract]) OR (Emergency Service, Medical[Title/Abstract])) OR (Medical Emergency Service[Title/ Abstract])) OR (Medical Emergency Services[Title/Abstract])) OR (Service, Medical Emergency[Title/Abstract])) OR (Services, Medical Emergency[Title/ Abstract])) OR (Medical Services, Emergency [Title/Abstract])) OR (Emergency Medical Service[Title/ Abstract])) OR (Medical Service, Emergency[Title/Abstract])) OR (Service, Emergency Medical[Title/Abstract])) OR (Services, Emergency Medical[Title/ Abstract])) OR (Prehospital Emergency Care[Title/Abstract])) OR (Emergency Care, Prehospital[Title/Abstract])) OR (Emergicenters[Title/Abstract])) OR (Emergicenter[Title/Abstract])) OR (Emergency Care[Title/Abstract])) OR (Emergency Health Services[Title/ Abstract])) OR (Emergency Health Service[Title/Abstract])) OR (Health Service, Emergency[Title/Abstract])) OR (Health Services, Emergency[Title/ Abstract])) OR (Service, Emergency Health [Title/Abstract])) OR (Services, Emergency Health[Title/Abstract])) AND ((((national early warning scores[Title/ Abstract]) OR (NEWS[Title/Abstract])) OR (national early warning score[Title/ Abstract])) OR (risk score[Title/Abstract])))
AND ( ("Mortality" [Mesh]) OR ((((Mortalities[Title/Abstract]) OR (Death Rate[Title/Abstract])) OR (Death Rates[Title/Abstract])) OR (Rate, Death[Title/Abstract]))).

Participant or population: Patients in the prehospital setting.

Intervention: NEWS scores were used to predict short-term mortality in a prehospital setting.

\section{Comparator: Not applicable.}

Study designs to be included: Observational study.

Eligibility criteria: Inclusion criteria (1) The study recruited adult patients $(\geq 16$ years old) in the prehospital setting; (2) The study applied the NEWS or NEWS2; (3) Patients transported by ambulance in the prehospital setting; (4) The study outcomes of interest were admission to ICU, shortterm mortality or sepsis; Exclusion criteria: (1) The study population was children or pregnant; (2)Helicopter emergency service; (3)If studies used the same database, we included the study with the most patients and excluded the others.

Information sources: Four electronic databases will be searched (PubMed, Embase, Cochrane, Web of Science) for eligible studies published from January 2012 to February 2022. Full-length articles in English-language journals were eligible.

Main outcome(s): Short-term mortality (1day or 2-day or 7-day mortality).

Additional outcome(s): Admission to intensive care; sepsis.

Quality assessment / Risk of bias analysis: NOS (the Newcastle-Ottawa Scale) tool used to assess quality.

Strategy of data synthesis: Without heterogeneity, the data were combined with fixed effect, but with heterogeneity, the source of heterogeneity was 
investigated first, and then a reasonable way was selected for merging.If a large degree of heterogeneity is found, a metaregression analysis will be performed to identify potential sources of bias. Publication bias will also be assessed by Deek test with funnel plot asymmetry, and $P<0.05$ is considered statistically significant. All analyses will be performed using Revman 5.3 and Stata/MP16.0.

Subgroup analysis: A subgroup analysis of NEWS and NEWS2 cutoff values and their ability to predict outcomes (intensive care admission versus sepsis) is planned.

Sensitivity analysis: The sensitivity analysis will be repeated after studies with a high risk of bias are excluded.

Country(ies) involved: China.

Keywords: national early warning score; prehospital; mortality; ambulance.

Contributions of each author:

Author 1 - WAN WENLIN - Collecting data, statistical analysis, methodology, writingreview and editing.

Author 2 - LI LEIXI - Collecting data, statistical analysis and study design.

Author 3 - OU OU - Data curation and visualization.

Author 4 - CHEN BING - Investigation.

Author 5 - MA ZHIQUN - Supervision.

Author 6 - YUAN PING - Critical revision of the manuscript. 